\title{
Integration column: microwell arrays for mammalian cell culture
}

\author{
Mirren Charnley, ${ }^{a}$ Marcus Textor, ${ }^{* a}$ Ali Khademhosseini ${ }^{* b}$ and Matthias P. Lutolf $* c$
}

DOI: $10.1039 / b 918172 p$

\begin{abstract}
Microwell arrays have emerged as robust and versatile alternatives to conventional mammalian cell culture substrates. Using standard microfabrication processes, biomaterials surfaces can be topographically patterned to comprise high-density arrays of micron-sized cavities with desirable geometry. Hundreds to thousands of individual cells or cell colonies with controlled size and shape can be trapped in these cavities by simple gravitational sedimentation. Efficient long-term cell confinement allows for parallel analyses and manipulation of cell fate during in vitro culture. These live-cell arrays have already found applications in cell biology, for example to probe the effect of cell colony size on embryonic stem cell differentiation, to dissect the heterogeneity in single cell proliferation
\end{abstract}

\footnotetext{
${ }^{a}$ BioInterfaceGroup, Laboratory for Surface Science and Technology, Department of Materials, ETH Zurich, Switzerland. E-mail: marcus.textor@mat.ethz.ch; Fax: +41 (0)4463310 27;

Tel: + $41(0) 446326451$

${ }^{b}$ Harvard-MIT Division of Health Sciences and Technology, Department of Medicine. Brigham and Women's Hospital, Harvard Medical School, Cambridge, MA 02139, USA.E-mail:alik@rics.bwh.harvard.edu; Tel: + 1 617-388-9271

${ }^{c}$ Laboratory of Stem Cell Bioengineering and Institute of Bioengineering, Ecole Polytechnique Fédérale de Lausanne (EPFL), Switzerland.

E-mail: matthias.lutolf@epfl.ch;

Fax: + $41(0) 2169396$ 65;

Tel: + $41(0) 216931876$
}

kinetics of neural or hematopoietic stem/progenitor cell populations, or to elucidate the role of cell shape on cell function. Here, we highlight the key applications of these platforms, hopefully inspiring biologists to apply these systems for their own studies.

\section{Introduction}

In vitro cell culture is traditionally performed on ensembles of thousands of cells seeded on a flat substrate of a relatively 'large' (i.e. millimetre- to centimetre-scale) cell culture well. Single cell behavior in such a configuration is averaged over the entire population. This experimental approach is adequate in most cases, since many in vitro assays require large numbers of cells. However, not all cell populations can be considered homogeneous, and knowledge on the behavior of an individual cell can often be of critical importance. A point in case are primary adult stem cells. These cells are often rare and can only be isolated with limited purity, even when the most stringent isolation schemes are utilized. Since the phenotypes of stem cells are hardly any different from those of their already partially differentiated progenitor cells, averaging the behavior of individual cells over the entire population, such as changes in cell cycle kinetics or self-renewal/differentiation, is problematic. For example, since in many cases adult stem cells grow significantly slower than progenitor cells or are even non-dividing, stem cell behavior is often buried within the behavior of overgrowing progenitor cells.

Unicellular systems employing standard multiwell cell culture plates, such as 96-well plates, allow cells to be analyzed and followed over time at the single cell level as clones, but require relatively large amounts of expensive cell culture medium components and are highly inefficient. Generally, they lack throughput, and cell tracking by microscopy can be cumbersome as the movement of a single cell on a large flat substrate is not restricted, often resulting in a loss of the single cell from a microscopic field of view. Therefore, many experimental in vitro paradigms are essentially 'black boxes' when it comes to enhancing our understanding of single cell behaviors.

The limitations of conventional cell culture systems in conducting efficient and high-throughput single cell experiments has spurred the development of ever-evolving engineered cell culture platforms. Sophisticated systems such as microfluidic valves, optical tweezers, dielectrophoresis (DEP) or acoustic waves allow the efficient trapping and manipulation of single cells (e.g.ref. 1-10). However, although many of these elegant systems work well for single cell studies, they are not yet suitable for the everyday usage in cell biology labs. Up until now, they have

\section{Insight, innovation, integration}

Using straightforward microfabrication processes, biomaterials surfaces can be topographically structured to comprise high-density arrays of micron-sized cavities, termed 'microwell arrays'. These substrates are, for example, ideally suited to culture and probe the fate of large numbers of trapped single cells, such as rare and heterogeneous stem cells. Here we highlight the key applications of these platforms, hopefully inspiring biologists to apply these systems for their own studies. 
been rather complicated, often not compatible with existing laboratory techniques (such as hand pipetting) and instrumentation (such as microscopy stages or plate readers).

Arguably one of the most 'simple' yet robust families of platforms to analyze populations of single cells in medium to high throughput are microwell arrays (Fig. 1). Microwell arrays are topographically structured surfaces that comprise a high density (i.e. hundreds to thousands $/ \mathrm{cm}^{2}$ ) of micron-sized cavities of desirable geometry. These substrates can be fabricated using standard microfabrication processes such as photolithography. ${ }^{11}$ Briefly, in photolithography, a 'photoresist' is coated on a silicon substrate, and a photomask placed in contact with the photoresist, which is then exposed to UV light and locally crosslinked through the mask. Next, an organic solvent is used to remove the uncrosslinked photoresist, producing a so-called 'master' that consists of a silicon wafer with micron-sized features of the photoresist. These features are of complementary topography of the desired microwell substrate. Finally, a precursor of poly(dimethylsiloxane) (PDMS), an elastomer, can be poured over this master, thermally cured and peeled off to produce a transparent PDMS microwell substrate. The latter is often used directly for cell culture, which is an attractive approach due to its simplicity. However, PDMS is hydrophobic and known to strongly adsorb proteins such as growth factors from the cell culture medium, which can be a problem. Alternatively, having the inverted topography of a microwell array (i.e. an array of pillars), a PDMS master can be used as a stamp to micropattern soft materials such as hydrogels (Fig. 1A), which are then used as non-adhesive microwell arrays with biomimetic physicochemical properties.

Microwell arrays thus allow thousands of single cells to be randomly captured by sedimentation (via gravity) at the

A
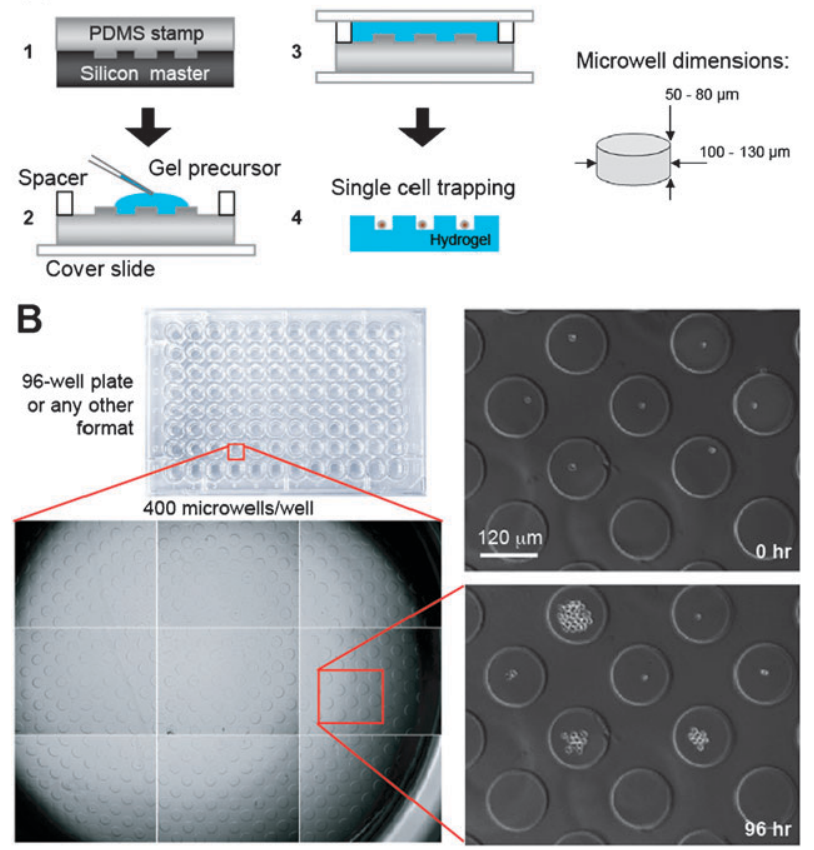

Fig. 1 Example of a microwell array platform based on soft hydrogels to systematically explore the function of single cells in high-throughput. (A) Overview of the multistep process to fabricate hydrogel microwell arrays. A PDMS stamp containing an array of micropillars is cast on a silicon master (step 1). This stamp is used as a template to crosslink a poly(ethylene glycol) (PEG) gel containing the complementary microwell array topography (steps 2 and 3). Upon swelling and washing, the hydrogel surface is used to trap large numbers of individual cells (step 4). Typical dimensions of the microwells are indicated on the right. (B) Hydrogel microwell arrays can be placed on the bottom of any standard well plate (here: 96-well) to culture single cells (here: mouse hematopoietic stem cells) and, for example, track their behavior by time-lapse videomicroscopy over many days. Adapted with permission from ref. 72. Copyright 2009 Royal Society of Chemistry.

bottom of the microwell cavities within a few minutes and analyzed over time in cell culture (Fig. 1B). ${ }^{12}$ Due to the stochastic nature of cell capturing, the experimenter ends up with an initial distribution of trapped cell numbers per microwell that is highly dependent on cell seeding density. Studies using single cell array systems (e.g.ref. 13) have shown that the histograms of numbers of trapped cells per microwell matches a Poisson distribution. The maximal occupancy of wells by single cells (and not doublets or triplets etc.) is generally around $30-40 \%$. Notably, microwells containing multiple cells at the onset of an experiment can be eliminated retrospectively from the analysis. Conversely, including microwells that host more than one cell at the onset of an experiment, it may be of interest to assess cell behavior as a function of the exact initial cell number. ${ }^{12}$ It should also be pointed out that in most microwell arrays, all trapped cells share the same medium, much like in a standard culture plate. That is, individual clones likely influence each other by secreting paracrine signaling factors.

Regardless of these limitations, due to their ease of fabrication and application, microwell arrays are increasingly being used for mammalian cell culture. We believe that three main types of applications are emerging (Fig. 2): (i) the engineering of controlled 'quasi-3D' single cell microenvironments mimicking essential features of the native 3D extracellular milieu (Fig. 2A), (ii) the fabrication of controlled cell aggregates ('colonies'), for example for stem and cancer cell biology as well as drug screening (Fig. 2B), and (iii) dynamic high-throughput single cell analyses using live-cell microscopy (Fig. 2C), which is particularly interesting for rare and heterogeneous cell populations such as stem cells. In this review we discuss these applications by way of specific examples and we outline future opportunities for the use of microwell platforms.

\section{Microwells as tunable artificial microenvironments for single cells}

It is widely known that properties of a cell's microenvironment play an essential role in governing cell behaviour. ${ }^{14}$ 

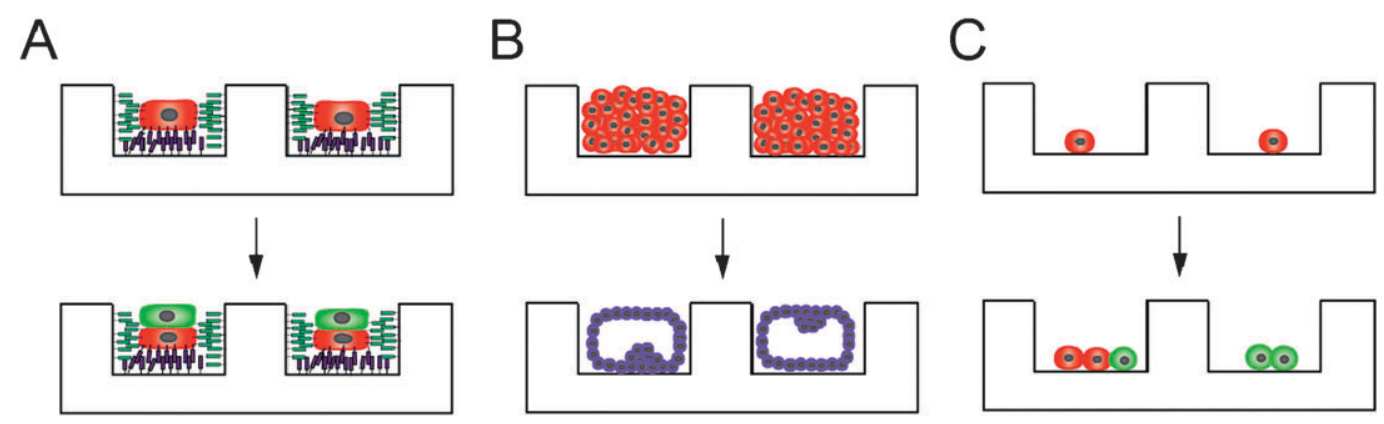

Fig. 2 Key applications of microwell array platforms in mammalian cell biology. (A) Engineering of controlled single cell microenvironments, mimicking key features of the native 3D milieu. (B) Generation of controlled cell aggregates ('colonies'). (C) Dynamic studies of changes in single stem cell behavior of heterogeneous cell populations.

Much of our understanding of cell physiology has been derived from cell culture studies performed on flat and rigid $2 \mathrm{D}$ substrates, typically composed of tissue culture polystyrene (TCPS) coated with a mixture of proteins, normally non-specifically adsorbed from the media. The cell is therefore exposed to a complex and somewhat ill-defined environment, which is a far cry from the milieu experienced in an in vivo context. ${ }^{15}$ In particular, such a 2D cell culture environment is highly heterogeneous, both at the cellsubstrate interface and in the number of cell-cell contacts experienced by the cell. Furthermore, the morphology of the cell can be dramatically influenced by the dimensionality of its environment. In contrast to $3 \mathrm{D}$, on flat substrates, adherent cells will become highly spread with numerous stretched stress fibres extending across the length of the cell.

Unsurprisingly, given the complexity of the system, in vitro behaviors of cells cultured in $2 \mathrm{D}$ often lack the ability to predict in vivo responses, such as the biocompatibility of implanted materials, where a range of materials generated similar responses in vivo (e.g. fibrous capsule formation) regardless of the in vitro behavior. ${ }^{16-18}$ Previous research also highlights, for example, the difficulty of $2 \mathrm{D}$ cell culture to predict the drug responsiveness of both cancer ${ }^{19,20}$ and liver cells. ${ }^{21}$

Although more physiologically relevant, the tools currently available for the study of cells in 3D environments such as in biopolymer gels (e.g. collagen or Matrigel $^{\mathrm{TM}}$ ) do not allow shape control of individual cells and cannot be used to make direct comparisons with results on
2D substrates. Furthermore, the available 3D tools do not allow the independent or homogenous modification of shape and substrate rigidity, making it difficult to decouple the effects of these environmental parameters. Finally, the imaging of cells cultured within $3 \mathrm{D}$ matrices can be laborious. As a consequence, the reasons for the differences in behavior between $2 \mathrm{D}$ and $3 \mathrm{D}$ cultures are poorly understood, and the relationships between dimensionality, matrix rigidity and cell shape are not known.

The use of microwells as engineered microenvironments to probe single cell behaviour is therefore an attractive alternative (Fig. 2A and 3). Microwells, in principle, allow the control of rigidity and cell shape, and provide a 'quasi-3D' environment that can be used to study the effects of these parameters nearly independently from each other. Obtained data should be directly comparable to those observed on 2D surfaces, since in both cases, cells are in contact with planar surfaces. Therefore, such cell culture platforms can be used to gain insight into how physical cues, in concert with biochemical signals, regulate cell behaviour.

\section{Engineering microwells as 2D single cell microenvironments}

Innovative approaches to trap single cells in microwells have been developed and optimized for a high rate of well occupancy by single cells, ${ }^{22-26}$ allowing for single cell analyses and retrieval using techniques such as fluorescence microscopy, capillary electrophoresis ${ }^{22}$ and laser capture microdissection. ${ }^{25}$ As pointed out above, such microwells can be fabricated from a variety of materials, typically PDMS or PEG hydrogels. Photolithographic patterning of PEG hydrogels onto glass can, for example, be utilized to form dense arrays of micrometre-sized wells composed of PEG hydrogel walls on glass substrates coated with either collagen or other cell-adhesive signals. ${ }^{25-27}$ Other groups have directly used PDMS to create microwells via soft lithography, resulting in either microwell chips composed of PDMS, ${ }^{23,24,28,29}$ or a PDMS stamp that was used to create microwells in the material of choice. ${ }^{30-32}$ These techniques have been successfully used to produce single cell arrays for a variety of cell types, including lymphocytes, ${ }^{25}$ fibroblasts, ${ }^{24}$ hepatocytes ${ }^{27}$ epithelial ${ }^{33}$ and endothelial cells. ${ }^{23,28,29}$ Studies typically focused on the analysis of cell attachment, ${ }^{24}$ morphology and spreading, ${ }^{22,23}$ viability, ${ }^{26}$ and cytoskeleton formation of cells cultured within microwells. ${ }^{30}$ However, of the microwell arrays just described, few have been used to study the effect of the microenvironment on single cell behaviour in a reductionist quasi-3D environment. There are nevertheless a few notable exceptions.

Nelson and Chen, for example, developed an approach for the study of cell-cell signalling by fabricating micropatterned quasi-3D substrates. ${ }^{31}$ These consisted of bowtie-shaped fibronectin islands on glass and agarose walls with an area of $750 \mu \mathrm{m}^{2}$. When cells were seeded on the substrate, pairs of cells spread to fill the adhesive area, with one cell on each side of the central constriction. Thus, this platform allowed cell shape to be controlled and decoupled from the formation of cell-cell contacts in order to independently 


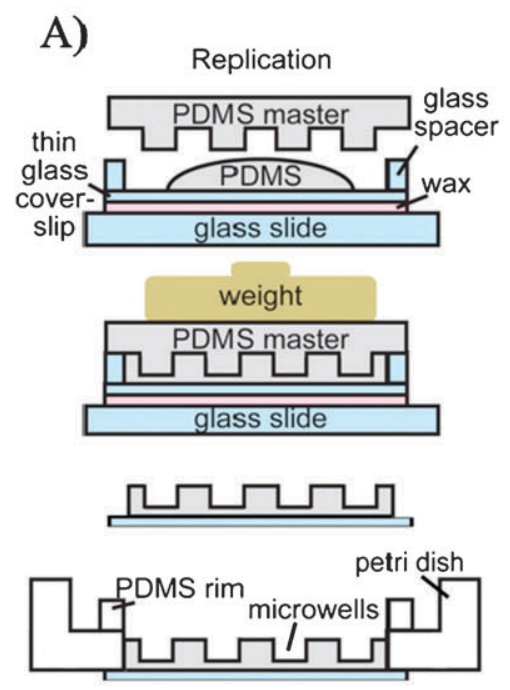

B)

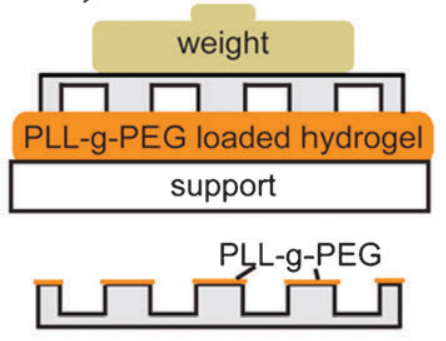

fibronectin solution
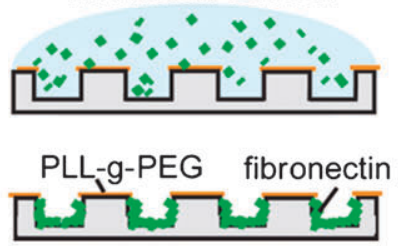

C)
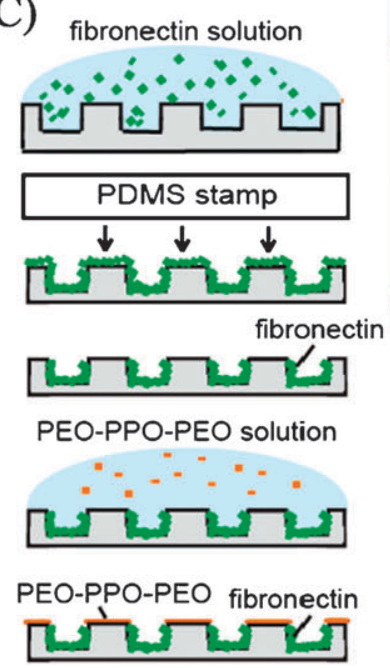
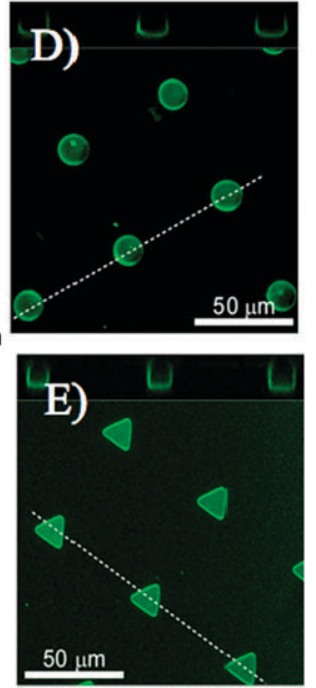

Fig. 3 (A) Outline of the replication techniques used to produce microwells in thin films of PDMS. A thin glass coverslip is glued onto a glass specimen slide between which the PDMS was cast. After curing, the master is removed, leaving behind a thin PDMS film bound to the coverslip, which is then glued to the bottom of a Petri dish into which a hole had been previously drilled. (B) The microwell plateau surface is passivated by inverted microcontact printing of PLL- $g$-PEG using a polyacrylamide stamp. The sample is then exposed to a fibronectin solution or a phospholipid vesicle solution and rinsed. (C) Alternatively, microwells can be coated with fibronectin, which is subsequently removed from the plateau using subtractive microcontact printing with a glutaldehdye-functionalised PDMS stamp. The plateau is finally passivated by exposure to pluronics solution. CLSM images showing (D) fluroescently labeled fibronectin or (E) labeled lipid bilayers that are only able to adsorb inside a microwell and no fibronectin or vesicle adsorbed onto the plateau. Adapted from ref. 29. Copyright 2007 Royal Society of Chemistry.

study their effects on proliferation. The authors were indeed able to demonstrate that the presence of cell-cell contacts increased proliferation, and that this effect was mediated by a P13K-dependent pathway, as opposed to paracrine signalling between cell pairs. In contrast, in a confluent layer of endothelial cells, proliferation was decreased, probably due to restricted cell spreading, which masked the increased proliferation stimulated by cell-cell contacts. This study therefore highlights the need for reductionist cell culture platforms that allow the effects of multiple parameters to be independently elucidated.

\section{Engineering microwells as near-physiological quasi-3D single cell microenvironments}

Most microwell platforms described in the literature consist either of an adhesive base and 'non-fouling' (i.e. inert) walls, ${ }^{26,31}$ or of microstructures greater than the size of a single cell, enabling spreading over the base of the well. ${ }^{23}$ When cells are cultured on these microwell chips, the cells will predominately interact with the base of the well and not the sides.
The cell will thus experience a 2D environment. Within the group of Marcus Textor, microwell culture chips were developed that constrain single cells such that the cell fills the entire well, and thereby experiences what can be considered as a 3D microenvironment (Fig. 3). ${ }^{28-30}$ PDMS microwells with volumes slightly larger than one cell were fabricated with different geometries (circles, triangles, rectangles, spindles, etc.), lateral dimensions from 81-900 $\mu^{2}$ and a depth of $10 \mu \mathrm{m}$. The upper surfaces of the wells were passivated via inverted microcontact printing with the cell-repellent co-polymer PLL-g-PEG, limiting cell adhesion to the wells. The inner surface was functionalized by physisorption with either fibronectin or lipid bilayers (Fig. 3B). ${ }^{29}$ Alternatively, the PDMS microwell chips were functionalized by physisorbing fibronectin onto the entire area of the microwells, which was subsequently removed from the plateau by subtractive microcontact printing with glutaldehdyefunctionalized PDMS (Fig. 3C). ${ }^{34}$ Pluronic $^{\mathrm{TM}}$, a poly(ethylene oxide)containing block copolymer, which suppresses protein adsorption, ${ }^{35}$ was then adsorbed onto these chips to passivate the plateau. This circumvented the issue of impaired fidelity of protein patterns as well as poor long-term stability that was reported for the PLL-g-PEG coating when used in $2 \mathrm{D}$ protein pattern applications. ${ }^{36}$ The fidelity of the microwell functionalization was maintained for up to 7 days in the culture of human bone marrow-derived mesenchymal progenitor cells, as demonstrated by the improved confinement of the cells within just the wells and not the plateau.

\section{Towards parsing cell shape, rigidity and dimensionality in single cell microwells}

Using these quasi-3D microwells, it was possible to decouple the effects of cell shape, substrate rigidity and dimensionality. ${ }^{37}$ Initially, the effect of limiting cell spreading on cell viability and actin formation was explored (Fig. 4). When cell spreading was constrained on micropatterned 2D surfaces, reduced viability and actin cytoskeleton formation was observed, similar to the results reported in the seminal study by Chen et al. ${ }^{38}$ In contrast, when cells were cultured in 3D microwells of the same substrate material and with similar spreading 
areas, they were both more viable and formed an actin cytoskeleton. The actin cytoskeleton was remarkably 3D-like, and prominent fibres were preferentially aligned along the long axis of the well. However, unlike cells cultured on larger 2D patterns, stretched stress fibres were not observed, similar to the lack of large actin bundles present in cells cultured in other 3D systems. ${ }^{39,40}$ This effect was observed only in relatively small microwells where the reduced spreading ensured that cells interacted with the walls of the wells in a 3D-like manner. In large wells (i.e. $>2500 \mu \mathrm{m}^{2}$ ), cells formed a monolayer similar to the 2D cultures, with stress fibres extending across the long axis of the cell and predominately concentrated at the cell-substrate interface. Therefore, a 3D arrangement of cell adhesive contacts allowed the cell to overcome limited-spreading-induced apoptosis, and stimulated actin cytoskeleton assembly even when cell spreading was limited to extents that did not promote actin cytoskeleton assembly in 2D.

One potentially confounding aspect of single cell arrays that should be considered here is the lack of cell-cell contacts present within these systems. The formation of cell-cell contacts is important for many cell functions such as proliferation, $^{31}$ cell polarization and directionality of cell migration, ${ }^{41}$ regulation of the sensitivity of the cell to the mechanical properties of its environment $^{42}$ and morphogenesis. ${ }^{43}$ Further, the reduced expression of proteins such as Cadherins that mediate cell-cell contacts can correlate with tumor formation and progression, ${ }^{44}$ conceivably through the loss of epithelial integrity and polarization. ${ }^{45}$ Therefore, an augmentation of microwell platforms towards a mimickry of this cell-cell crosstalk (Fig. 2A) would be of great benefit for many basic biological studies. Potential routes to obtain such spatially controlled protein patterning of microwells include the functionalization of wells with coatings that mimic certain aspects of cell contacts. ${ }^{4-49}$

Taken together, the above examples demonstrate that microwell arrays can be successfully exploited as reductionist microenvironments to independently control some parameters of the milieu surrounding a single cell. These platforms should expand the repertoire of tools for both fundamental biological studies as well as cell-based assays for drug screening.

\section{Microwell arrays for the control and manipulation of stem cell colony growth}

Over the past few years, microwell arrays have been increasingly used for controlling embryonic stem cell (ESC) behavior in vitro. ${ }^{50} \mathrm{~A}$ major reason for the use of microwells in ESC biology lies (i) in the heterogeneity of single cell developmental potential of common ESC populations, and (ii) in the influence of ESC colony size on selfrenewal and differentiation. Microwell arrays allow the growth of ESCs at the clonal level and in high-throughput, and they also can be used to produce ESC colonies, such as embryoid bodies (EBs), of well-controlled size and shape, potentially helping to overcome the aforementioned issues in in vitro ESC biology.

Conventional ESC aggregates are formed by scraping colonies off a culture surface. This results in predominantly disordered colonies, differing widely in size and shape. ${ }^{51}$ Accordingly, this heterogeneity is passed on to the differentiating aggregate. Consequently, the local microenvironment within or between aggregates is highly inconsistent. Microwell technology has been used to form controlled cell aggregates (Fig. 2B) from both mouse and human ESCs. In some of the early work, gelatin-coated microwell substrates were used to selectively adhere human ESCs to the bottom of the microwells to give rise to ESC aggregates. ${ }^{52}$ These aggregates maintained markers of pluripotency over an extended period of time. In follow-up work, feeder cells were seeded on microwell arrays and induced to form a monolayer that could be used for maintaining ESC aggregates in an undifferentiated-like state. $^{53}$ These co-cultures provided ESCs with
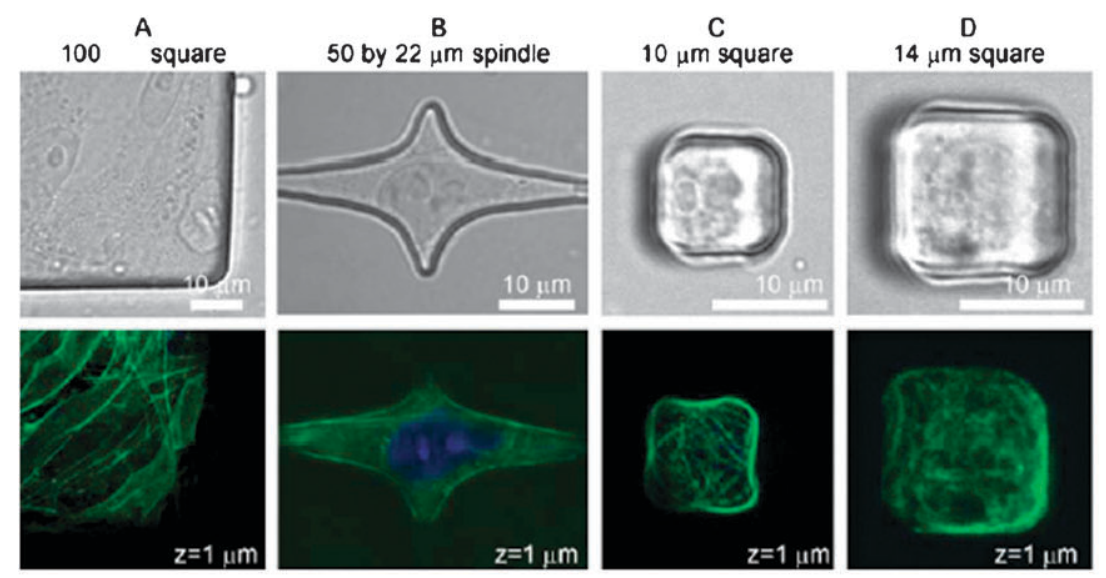

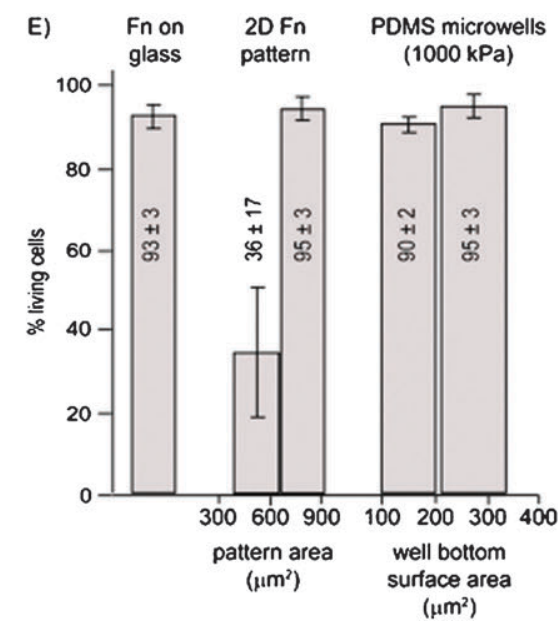

Fig. 4 Confocal scanning laser microscopy images show the nucleus (blue; ethidium homodimer) and actin (Phalloidin 488, green) of primary human endothelial cells cultured for 24 hours in a very large microwell (A), a spindle shaped microwell (B), and 10 (C) and $14 \mu \mathrm{m}$ (D) square microwells with adequately controlled 3D shape of the well. (E) The cell viability of primary human endothelial cells cultured for 24 hours was assessed after being cultured on 2D substrates, or within 3D microwells. Reduced viability was observed after culture on small patterns, in contrast to cells cultured within small microwells. Adapted from ref. 29. Copyright 2007 Royal Society of Chemistry. 
the necessary self-renewing signals generated by the feeder cells, while at the same time allowed for controlling the size of the resulting colonies.

\section{Microwell cultures to control embryoid body (EB) formation}

A major use of microwells for ESC cultures has been for controlling the formation of EBs. ${ }^{54} \mathrm{EBs}$ mimic some of the early stages of embryonic development and initiate the formation of various germ layers, which in vivo would give rise to an entire organism. Typically, EBs are generated using non-adhesive dishes; however, in these cultures, heterogeneous cell aggregates are formed. The existence of this heterogeneity has been shown to result in size-dependant differentiation. ${ }^{55}$ To alleviate this issue, methods such as hanging drop cultures have been developed. Hanging drops are made by inverting a drop of medium and aggregating the cells at the air-medium interface. The limitation of this technique is its low throughput, which can be readily overcome by microwell arrays.

For example, PEG hydrogel microwells have been used as a template for forming EBs. ${ }^{40,56}$ These templates create low shear stress regions to enable docking of the cells inside the microwells and subsequently can be used to form cell aggregates. ${ }^{57,58}$ Various parameters such as the size of the PEG macromers used to crosslink PEG microwell arrays can significantly influence the long-term response of
EBs in these cultures. Specifically, in culture conditions in which the microwells were made from relatively hydrophobic lower molecular weight PEG, cells tended to stick to the entire surfaces, whereas in cultures made from more hydrophilic higher molecular weight PEG, it was possible to maintain the non-adherent features of these microwells much longer. In addition, other techniques have been used to fabricate microwells for ESC cultures. ${ }^{59}$ For example, by selective deformation of a substrate to pressure, ${ }^{60,61}$ microwells with rounded shapes were produced that are suitable to generate EBs.

The size of EBs can be readily controlled by the microwell dimensions and has been shown to dramatically influence ESC differentiation (Fig. 5).
A
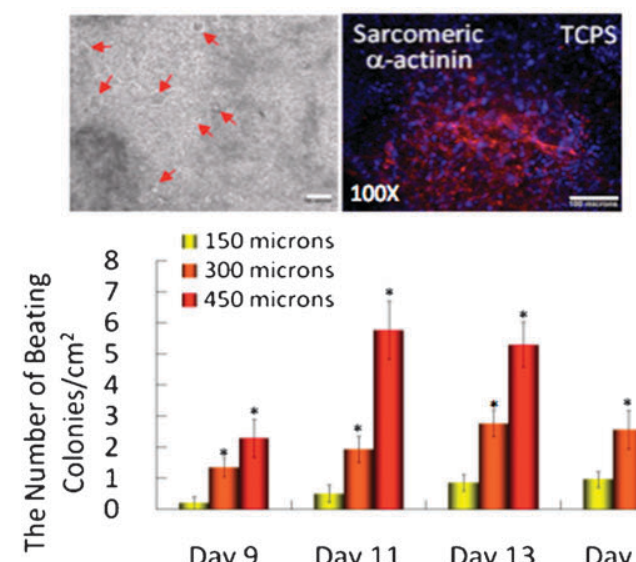

$\mathbf{C}_{(1)}$

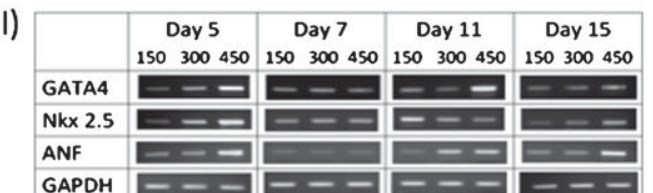

(II)

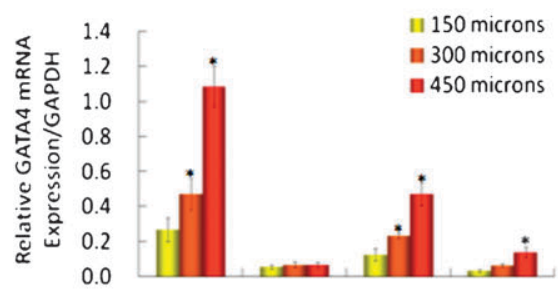

(III)

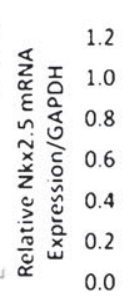

B
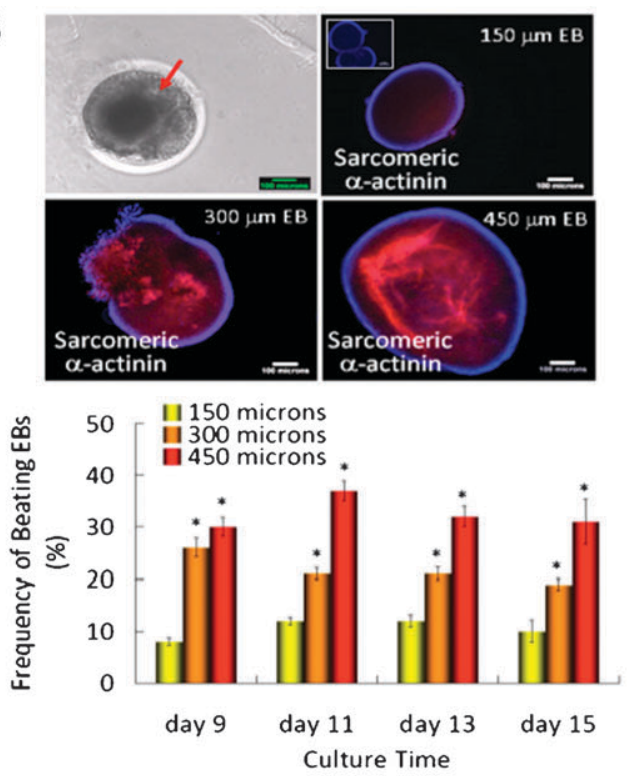

(IV)
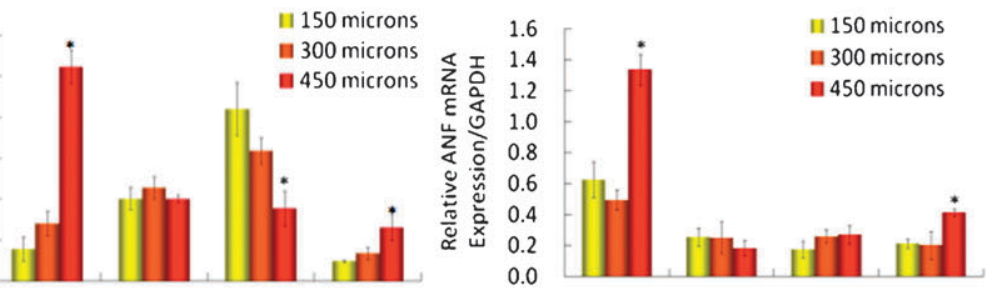

Fig. 5 EB size-mediated cardiogenic differentiation of ES cells. (A) Morphology and characterization of beating foci (red arrows) in EB outgrowths. Immunocytochemical characterization of cardiomyogenic differentiation and quantification of beating colonies from EB outgrowths that were replated from microwells. Sarcomeric $\alpha$-actinin expression (red) of EB outgrowths at day 15 of culture. Scale bars are $100 \mu \mathrm{m}$. (B) Morphology of beating EBs, immunocytochemical characterization of cardiomyogenic differentiation identified by sarcomeric $\alpha$-actinin and evaluation of beating EBs cultured in microwells. Inset for $150 \mu \mathrm{m}$ EB figure indicates control stained only with secondary antibody. Scale bars are $100 \mu \mathrm{m}$. (C) Time course of cardiomyogenic gene expression from EBs within microwells. (I) Gel pictures, (II) GATA4 mRNA expression, (III) Nkx2.5 mRNA expression, and (IV) ANF mRNA expression. Data shown as mean normalized mRNA expression intensity \pm SEM $(n=3$, * indicates $p<0.05$ compared to $150 \mu \mathrm{m}$ EB). Reprinted with permission from ref. 62. Copyright 2009 National Academy of Sciences, USA. 
The group of Peter Zandstra used PDMS microwell arrays (dimensions: 100, 200, 400 and 800 micron) inserted into 24-well plates for the production of hundreds to thousands of well-defined, spatially and temporally synchronised human EBs per $\mathrm{cm}^{2} .51$ They demonstrated that human ESC input composition and inductive environment could be manipulated to form large numbers of well-defined EBs exhibiting multi-lineage differentiation and substantially improved self-organization. Khademhosseini and colleagues specifically analyzed cardiac and endothelial cell differentiation from EBs and demonstrated that larger aggregates in microwells preferentially underwent cardiac differentiation, whereas those that were in smaller aggregates resulted in endothelial-like cells. ${ }^{62}$ Follow-up studies demonstrated that the morphogen Wnt11 was expressed at higher levels in larger aggregates and resulted in more cardiac differentiation, whereas Wnt5a was expressed in smaller aggregates and induced increased endothelial cell differentiation. Finally, it should be noted that other types of surface patterning approaches have also been successfully used to control ESC aggregation and to pattern ESCs on surfaces. ${ }^{63,64}$ These studies have consistently demonstrated that size control and its effect on regulating ESC fate decisions is an important consideration in ESC biology. ${ }^{65,66}$

\section{Interfacing microwell technologies with other technologies for the study of pluripotent stem cells}

An interesting aspect of microwells is that not only can they be used to control the size of cell aggregates, but they can also be easily integrated into other platforms. ${ }^{67}$ For example, it was demonstrated that cells can be seeded inside microwells hosted in microfluidic channels. $^{68}$ This should enable one to study the effects of various extrinsic factors on the cells within separated microfluidic channels.

The advent of new technologies to facilitate the development of cellular systems such as induced pluripotent stem (iPS) cells make the use of microwells even more appealing. ${ }^{69}$ In addition to directing ESC differentiation, it may be possible to use microwells to study and influence the reprogramming of desired cell types. Due to their small volumes and highly controllable environments, microwells should be attractive for creating scalable systems within which cell behavior can be controlled and tracked such that an array of cells can be exposed to different reprogramming factors. Thus, these approaches provide a powerful set of tools for controlling the ESC fate decisions.

\section{Analyses of the dynamics of single adult stem cell fate in microwell arrays}

Microwell array platforms are also well suited for the study of adult stem cell biology, since these cell populations are rare and known to be highly heterogeneous. Bhatia and colleagues reported a pioneering example of the use of a thoroughly characterized microwell array for adult stem cell biology. ${ }^{12}$ Microwells with diameters of $20-500$ microns were produced on glass coverslips at a density of $c a .10000$ microwells per slide, and applied to the culture and dynamic analyses of single adult (rat) progenitor cells isolated from the hippocampus. Automated live-cell microscopy was utilized to track the proliferation and death of several thousand single cells in culture over several days. These experiments revealed a marked heterogenetiy in proliferation kinetics between clones of the same population, and a small subpopulation (3-4\%) of highly proliferative clones. The authors further showed that a difference in starting numbers of cells per microwell (one, two or three cells) did not significantly influence proliferation kinetics.

In a related study, Cordey et al. reported the application of a PEG hydrogel microwell array platform ${ }^{32}$ to explore the development of single mouse neural stem/progenitor cells into multicellular aggregates, termed 'neurospheres'. ${ }^{70}$ Compared to the conventional neurosphere culture method on non-adherent flat plastic dishes, single NSC viability on soft hydrogels was reported to be two-fold higher. Effective confinement of single

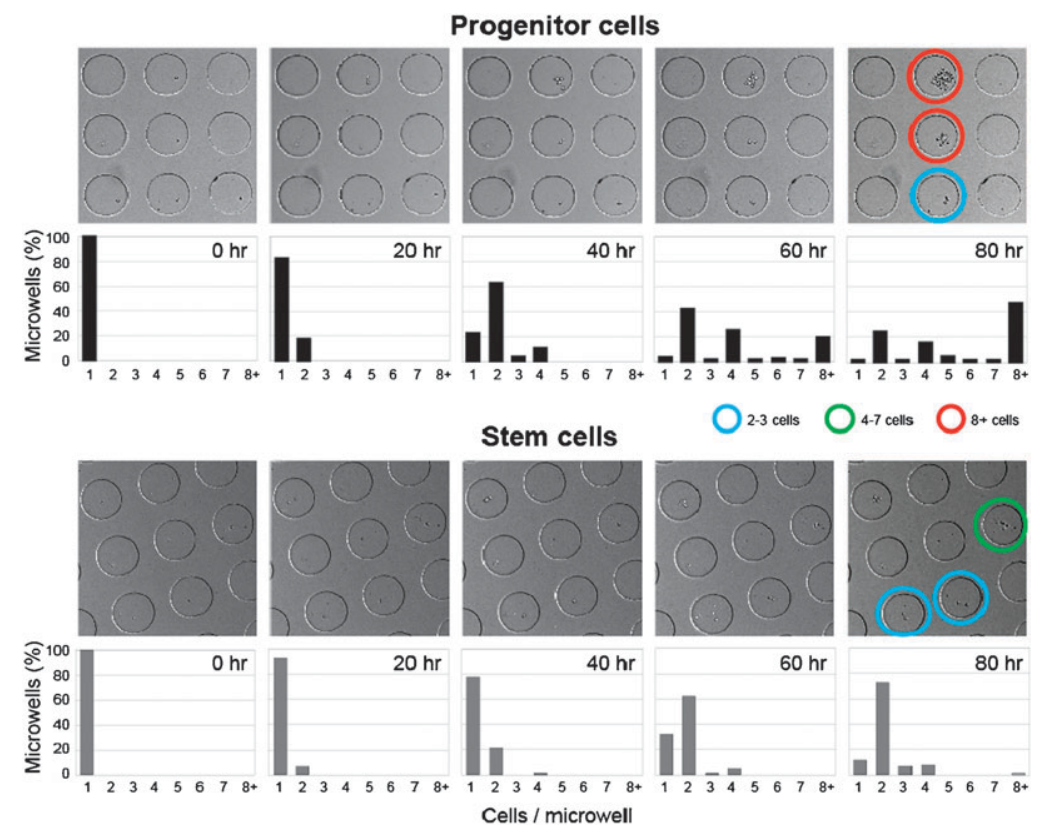

Fig. 6 Time-lapse videomicroscopy to quantify single hematopoietic stem and progenitor cell proliferation behavior. Progenitor cells (top panels) are highly proliferative, while stem cells (bottom panels) display slower proliferation kinetics. Coloured circles indicate microwells hosting clones that underwent variable numbers of divisions. Quantification of the distribution of cells per microwell at the indicated time point confirm visual differences: for example, $50 \%$ of all microwells comprised 8 or more cells in the progenitor population, while $70 \%$ of microwells of stem cells contained only 2 cells. Adapted with permission from ref. 72. Copyright 2009 Royal Society of Chemistry. 
proliferating cells to microwells led to neurosphere formation of vastly different sizes, in contrast to conventional neurosphere cultures in which slowly proliferating clones may be missed. Cells in microwell-cultured neurospheres showed a high percentage of stem/progenitor cell phenotype (Hes5 + Nestin + Beta3tubulin-) after one week in culture.

The in vitro behavior of single hematopoietic stem cells (HSC) has also been intensely investigated using microwell arrays. Dykstra and colleagues, for example, tracked the dynamic behavior of single mouse HSCs on PDMS microwell arrays and were able to correlate proliferative behavior in vitro with in vivo function, that is, the multilineage reconstitution of the blood of lethally irradiated mice. ${ }^{71}$ As a result of the achieved single cell resolution, these authors detected new hallmarks of proliferating HSCs, such as particular morphologies that are, at least to some extent, predictive of self-renewal divisions.

Using PEG hydrogel microwell arrays (Fig. 1B), Lutolf and Blau and colleagues expanded on these results, and, by purifying different stem and progenitor cell populations from the bone marrow of mice using fluorescenceactivated cell sorting, were able to show that individual stem cells possess significantly prolonged cell cycle times compared to progenitor cells when cultured on microwell arrays (Fig. 6). ${ }^{72}$ Using automated time-lapse microscopy, the kinetic proliferation profiles of single cells were quantified, for example, as the distribution of numbers of HSC progeny generated per microwell as a function of time (Fig. 6).

In an effort to elucidate the molecular signaling characteristics of HSC niches, the above authors went on to track the change in fate of single mouse HSCs in response to selected putative niche protein components. ${ }^{72}$ HSC behavior was analyzed in vitro by live-cell microscopy in combination with in vivo transplantation assays. To 'deconstruct' and mimic HSC niches, PEG hydrogel microwells were selectively functionalized at the bottom with regulatory proteins using a combination of soft lithography and microcontact printing (Fig. 7). Protein tethering was achieved by attaching a heterofunctional PEG linker to a protein of interest and then crosslinking this conjugate into the forming gel network (Fig. 7A). To ensure siteselectivity in protein immobilization, engineered Fc-chimeric proteins were linked on the gel surface via binding to an intermediate auxiliary protein, ProteinA, that contains four high-affinity binding sites ( $K_{\mathrm{a}}=10^{8}$ per mole) for the Fc-region of several immunoglobulins. Immunofluorescence microscopy indeed revealed that microcontact printed proteins, such as a BSA-FITC model protein were only localized at the bottom of the microwells (Fig. 7C). When ProteinA was used, Fc-chimeric proteins such as N-Cadherin were shown via immunostaining to be effectively tethered (Fig. 7D). This selective microwell modification was crucial to prevent the escape of trapped cells from microwells, particularly in the case of modification with adhesion proteins such as fibronectin. Using this artificial niche platform, the authors could demonstrate that single HSCs underwent self-renewal divisions in vitro in response to selected immobilized proteins. For example, a reduction in proliferation kinetics or an increase in asynchronous division of single HSCs in microwells in response to the protein Wnt3a or tethered N-Cadherin correlated well with subsequent serial long-term blood reconstitution in mice in vivo.

The group of Carsten Werner recently investigated the influence of spatial restriction and adhesive interactions on human CD133 + HSC fate decisions in vitro. ${ }^{73}$ Fibronectin-coated microwell arrays (Fig. 8) were prepared and single cell behavior on these substrates investigated. Proliferation and differentiation was shown to decrease when single HSCs were exposed to small microwells. Notably, single cell analysis of adherent cells revealed decreased DNA synthesis and higher levels of HSC marker expression inside the smaller cavities. These results thus suggested the spatial confinement
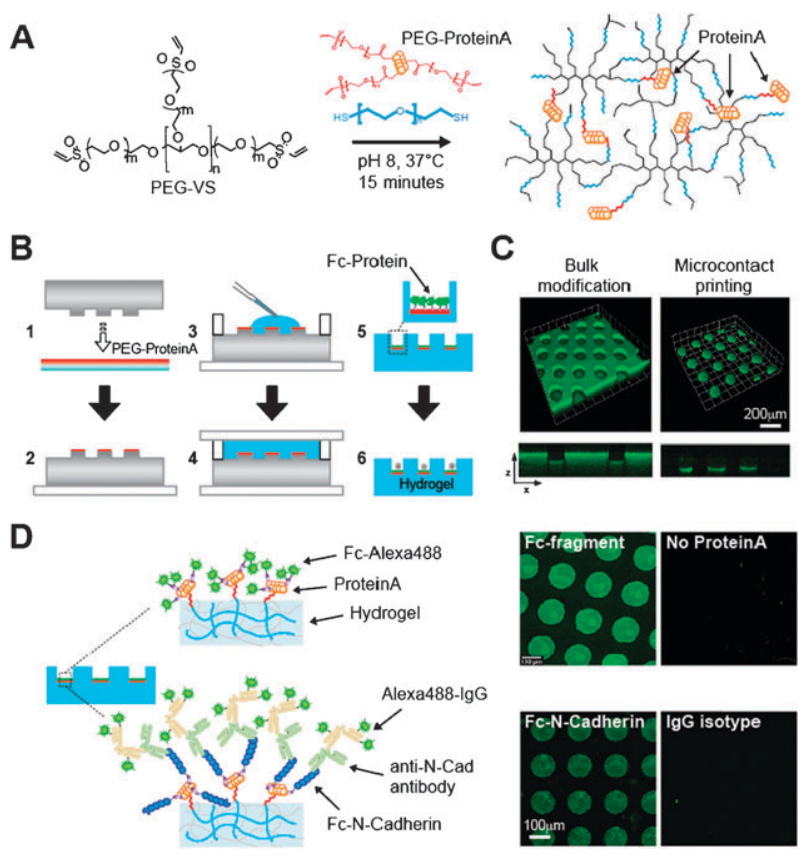

Fig. 7 Fabrication of hydrogel niches for single adult stem cells. (A) Covalent chemistry used to crosslink hydrogel networks functionalized with ProteinA. (B) Process to locally functionalize hydrogel microwell arrays with proteins: PEG-functionalized ProteinA was adsorbed onto the posts of the PDMS stamp (steps 1 and 2) and the hydrogel polymerized onto the ProteinA/PDMS (steps 3 and 4), transferring both the topographic pattern and protein pattern onto the gel surface. (C) Bulk versus local patterning of gel surfaces using FITC-BSA as model protein (3D confocal micrographs of projection of 84 stacks acquired at a constant slice thickness of $1.8 \mu \mathrm{m}$ ). (D) Binding of Fc-ligand and Fc-chimeric N-Cadherin onto immobilized ProteinA revealed using immunostaining (negative controls: microwell arrays not tethered with Protein A or treated with isotype control primary antibody). Reprinted with permission from ref. 72. Copyright 2009 Royal Society of Chemistry. 

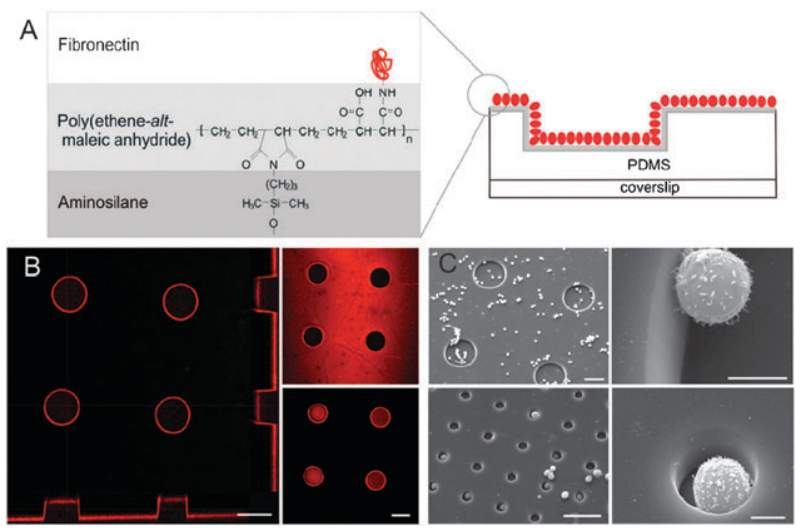

Fig. 8 Protein immobilization on a microstructured surface. (A) After covalent coupling of poly(ethene-alt-maleic anhydride) to the silicone surface, fibronectin is covalently attached via its lysine side chain to the anhydride moieties. The surface quality was assayed using 3D imaging of fluorescent-labeled fibronectin by confocal laser scanning microscopy (B) and scanning electron microscopy (C). HSC adhesion in the microcavities is visualized in (C). Scale bars: (B) $40 \mu \mathrm{m}$, (C) $50 \mu \mathrm{m}$ (left) and $5 \mu \mathrm{m}$ (right). Adapted with permission from ref. 73. Copyright 2009 Royal Society of Chemistry.

of human HSCs in fibronectin-coated microwells as a possible means to maintain a quiescent stem cell state, one of the hallmarks of long-term HSC in their in vivo niches.

Taken together, the above dynamic studies on adult stem cells in microwells highlight the relevance of single cell resolution in in vitro cell assays. Microwell arrays could be applied to many other stem cell populations to help shedding light on the mechanisms of stem cell regulation, knowledge which is crucial for the manipulation of these stem cells in clinical settings.

\section{Conclusions}

Microwell arrays, fabricated from many types of biomaterials including glass, bulk polymers or polymer hydrogels, have emerged as versatile alternatives to conventional cell culture substrates. Because microwell arrays are relatively simple, and compatible with existing laboratory techniques and instrumentation, they are well suited for everyday usage in any research laboratory.

As discussed above, microwells engineered as single cell microenvironments (Fig. 2A) could potentially offer unique opportunities to manipulate cell function. Here, the challenges will be to further develop them towards a clean parsing of the many parameters that control cell fate in 3D (i.e. microwell shape, matrix stiffness, protein composition, dose and spatial arrangement, matrix proteolytic degradation, and porosity, etc.). If this challenge is solved, these platforms will surely facilitate many fundamental biological studies in the near future.

Microwell arrays should also be attractive as tools to speed up drug discovery. ${ }^{74-76}$ One application of interest is the use of cell spheroids (Fig. 2B), such as those made from embryonic stem cells (as discussed above), hepatocytes, ${ }^{77}$ mesenchymal stem cells, ${ }^{78}$ neural stem cells $^{70}$ or tumor cells. ${ }^{79}$

However, it should be noted that microwells do not provide any new functionality compared to conventional cell culture systems. Microwell cultures are static, limiting their possibilities to actively manipulate trapped single cells, for example to conduct medium changes or to temporally control the exposure of cells to certain mitogenic stimuli such as is possible for example with microfluidic-based culture systems such as microchannel devices. ${ }^{80}$ Thus, as 'simple' high-throughput single cell platforms (Fig. 2C), microwell arrays, similar to high-density plastic well plates (1536-well plates) simply bridge the gap between standard well formats with low throughput, such as 96-wells, and more sophisticated platforms such as microfluidics.

Nevertheless, we strongly believe that microwell arrays are poised to replace to some extent conventional cell culture paradigms, particularly when single cell resolution is required.

\section{References}

1 H. Andersson and A. van den Berg, Curr. Opin. Biotechnol., 2004, 15, 44-49.

2 R. M. Johann, Anal. Bioanal. Chem., 2006, 385, 408-412.

3 J. Voldman, Annu. Rev. Biomed. Eng., 2006, 8, 425-454.

4 J. S. Marcus, W. F. Anderson and S. R. Quake, Anal. Chem., 2006, 78, 3084-3089.

5 D. R. Albrecht, G. H. Underhill, T. B. Wassermann, R. L. Sah and S. N. Bhatia, Nat. Methods, 2006, 3, 369-375.

6 N. Mittal, S. Flavin and J. Voldman, J. Vis. Exp., 2007, 318.

7 M. He, J. S. Edgar, G. D. Jeffries, R. M. Lorenz, J. P. Shelby and D. T. Chiu, Anal. Chem., 2005, 77, 1539-1544.

8 T. Braschler, N. Demierre, E. Nascimento, T. Silva, A. G. Oliva and P. Renaud, Lab Chip, 2008, 8, 280-286.

9 D. Di Carlo, Y. Liz and L. P. Lee, Lab Chip, 2006, 6, 1445-1449.

$10 \mathrm{~W}$. Tan and S. Takeuchi, Proc. Natl. Acad. Sci. U. S. A., 2007, 104, 1146-1151.

11 D. B. Weibel, W. R. DiLuzio and G. M. Whitesides, Nat. Rev. Microbiol., 2007, 5, 209-218.

12 V. I. Chin, P. Taupin, S. Sanga, J. Scheel, F. H. Gage and S. N. Bhatia, Biotechnol. Bioeng., 2004, 88, 399-415.

13 K. Ino, M. Okochi, N. Konishi, M. Nakatochi, R. Imai, M. Shikida, A. Ito and H. Honda, Lab Chip, 2008, 8, 134-142.

14 K. M. Yamada and E. Cukierman, Cell, 2007, 130, 601-610.

15 D. G. Castner and B. D. Ratner, Surf. Sci., 2002, 500, 28-60.

16 C. Gretzer, L. Emanuelsson, E. Liljensten and P. Thomsen, J. Biomater. Sci. Polym. Ed., 2006, 17, 669-687.

17 L. P. Tang, Y. L. Wu and R. B. Timmons, J. Biomed. Mater. Res., 1998, 42, 156-163.

18 W. G. Brodbeck, G. Voskerician, N. P. Ziats, Y. Nakayama, T. Matsuda and J. M. Anderson, J. Biomed. Mater. Res., 2003, 64A, 320-329.

19 J. M. Padron, C. L. van der Wilt, K. Smid, E. Smitskamp-Wilms, H. H. J. Backus, P. E. Pizao, G. Giaccone and G. J. Peters, Crit. Rev. Oncol. Hematol., 2000, 36, 141-157.

20 B. A. Teicher, T. S. Herman, S. A. Holden, Y. Y. Wang, M. R. Pfeffer, J. W. Crawford and E. Frei, Science, 1990, 247, 1457-1461.

21 Y. C. Toh, C. Zhang, J. Zhang, Y. M. Khong, S. Chang, V. D. Samper, D. van Noort, D. W. Hutmacher and H. R. Yu, Lab Chip, 2007, 7, 302-309.

22 K. Leong, A. K. Boardman, H. Ma and A. K. Y. Jen, Langmuir, 2009, 25, 4615-4620.

23 E. Ostuni, C. S. Chen, D. E. Ingber and G. M. Whitesides, Langmuir, 2001, 17, 2828-2834.

24 J. R. Rettig and A. Folch, Anal. Chem., 2005, 77, 5628-5634.

25 A. Revzin, K. Sekine, A. Sin, R. G. Tompkins and M. Toner, Lab Chip, 2005, 5, 30-37.

26 A. Revzin, R. G. Tompkins and M. Toner, Langmuir, 2003, 19, 9855-9862. 
27 A. Revzin, P. Rajagopalan, A. W. Tilles, F. Berthiaume, M. L. Yarmush and M. Toner, Langmuir, 2004, 20, 2999-3005.

28 M. R. Dusseiller, M. L. Smith, V. Vogel and M. Textor, Biointerphases, 2006, 1, P1-P4.

29 M. Ochsner, M. R. Dusseiller, H. M. Grandin, S. Luna-Morris, M. Textor and M. L. Smith, Lab Chip, 2007, 7, 1074-1077.

30 M. R. Dusseiller, D. Schlaepfer, M. Koch, R. Kroschewski and M. Textor, Biomaterials, 2005, 26, 5917-5925.

31 C. M. Nelson and C. S. Chen, FEBS Lett., 2002, 514, 238-242.

32 S. Kobel, M. Limacher, S. Gobaa, T. Laroche and M. P. Lutolf, Langmuir, 2009, 25, 8774-8779.

33 M. R. Dusseiller, D. Schlaepfer, M. Koch, R. Kroschewski and M. Textor, Biomaterials, 2005, 26, 5917-5925.

34 M. Rottmar, M. Håkanson, M. L. Smith and $\mathrm{K}$. Maniura, submitted.

35 J. T. Li and K. D. Caldwell, Colloids Surf., B, 1996, 7, 9-22.

36 J. W. Lussi, D. Falconnet, J. A. Hubbell, M. Textor and G. Csucs, Biomaterials, 2006, 27, 2534-2541.

37 M. Ochsner, M. Textor, V. Vogel and M. L. Smith, submitted.

38 C. S. Chen, M. Mrksich, S. Huang, G. M. Whitesides and D. E. Ingber, Science, 1997, 276, 1425-1428.

39 K. A. Beningo, M. Dembo and Y. I. Wang, Proc. Natl. Acad. Sci. U. S. A., 2004, 101, 18024-18029.

40 J. M. Karp, J. Yeh, G. Eng, J. Fukuda, J. Blumling, K. Y. Suh, J. Cheng, A. Mahdavi, J. Borenstein, R. Langer and A. Khademhosseini, Lab Chip, 2007, 7, 786-794.

41 R. A. Desai, L. Gao, S. Raghavan, W. F. Liu and C. S. Chen, J. Cell Sci., 2009, 122, 905-911.

42 T. Yeung, P. C. Georges, L. A. Flanagan, B. Marg, M. Ortiz, M. Funaki, N. Zahir, W. Y. Ming, V. Weaver and P. A. Janmey, Cell Motil. Cytoskeleton, 2005, 60, 24-34.

43 L. Larue, M. Ohsugi, J. Hirchenhain and R. Kemler, Proc. Natl. Acad. Sci. U. S. A., 1994, 91, 8263-8267.

44 G. S. Du, J. M. Wang, J. X. Lu, Q. Li, C. Q. Ma, J. T. Du and S. Q. Zou, Ann. Surg. Oncol., 2009, 16, 1578-1586.

45 M. V. Fournier, J. E. Fata, K. J. Martin, P. Yaswen and M. J. Bissell, Cancer Res., 2009, 69, 4545-4552.
46 A. L. DeMond, K. D. Mossman, T. Starr, M. L. Dustin and J. T. Groves, Biophys. J., 2008, 94, 3286-3292.

47 M. Lambert, F. Padilla and R. M. Máege, J. Cell Sci., 2000, 113, 2207-2219.

48 K. Mossman and J. Groves, Chem. Soc. Rev., 2007, 36, 46-54.

49 J. Tsai and L. Kam, Biophys. J., 2009, 96, L39-L41.

50 A. Khademhosseini, R. Langer, J. Borenstein and J. P. Vacanti, Proc. Natl. Acad. Sci. U. S. A., 2006, 103, 2480-2487.

51 M. D. Ungrin, C. Joshi, A. Nica, C. Bauwens and P. W. Zandstra, PLoS One, 2008, 3, 1565.

52 J. C. Mohr, J. J. de Pablo and S. P. Palecek, Biomaterials, 2006, 27, 6032-6042.

53 A. Khademhosseini, L. Ferreira, J. Blumling 3rd, J. Yeh, J. M. Karp, J. Fukuda and R. Langer, Biomaterials, 2006, 7, 5968-5977.

54 L. G. Villa-Diaz, Y. S. Torisawa, T. Uchida, J. Ding, N. C. Nogueira-deSouza, K. S. O'Shea, S. Takayama and G. D. Smith, Lab Chip, 2009, 9, 1749-1755.

55 C. Alperin, P. W. Zandstra and K. A. Woodhouse, Front. Biosci., 2007, 12, 3694-3712.

56 N. Peppas, J. Z. Hilt, A. Khademhosseini and R. Langer, Adv. Mater., 2006, 18, 1345-1360.

57 A. Khademhosseini, J. Yeh, S. Jon, G. Eng, K. Y. Suh, J. A. Burdick and R. Langer, Lab Chip, 2004, 4, 425-430.

58 H. C. Moeller, M. K. Mian, S. Shrivastava, B. G. Chung and A. Khademhosseini, Biomaterials, 2008, 29, 752-763.

59 C. S. Chen, J. Pegan, J. Luna, B. Xia, K. McCloskey, W. C. Chin and M. Khine, J. Vis. Exp., 2008.

60 N. C. Rivron, J. Rouwkema, R. Truckenmuller, M. Karperien, J. De Boer and C. A. Van Blitterswijk, Biomaterials, 2009, 30, 4851-4858.

61 R. Truckenmüller, S. Giselbrecht, C. van Blitterswijk, N. Dambrowsky, E. Gottwald, T. Mappes, A. Rolletschek, V. Saile, C. Trautmann, K. F. Weibezahn and A. Welle, Lab Chip, 2008, 8, 1570-1579.

62 Y. S. Hwang, B. G. Chung, D. Ortmann, N. Hattori, H. C. Moeller and K. A, Proc. Natl. Acad. Sci. U. S. A., 2009, 106, 16978-16983.

63 C. L. Bauwens, R. Peerani, S. Niebruegge, K. A. Woodhouse, E. Kumacheva, M. Husain and P. W. Zandstra, Stem Cells, 2008, 26, 2300-2310.
64 S. Jinno, H. C. Moeller, C. L. Chen, B. Rajalingam, B. G. Chung, M. R. Dokmeci and A. Khademhosseini, J. Biomed. Mater. Res. A, 2008, 86, 278-288.

65 J. Park, C. H. Cho, N. Parashurama, Y. Li, F. Berthiaume, M. Toner, A. W. Tilles and M. L. Yarmush, Lab Chip, 2007, 7, 1018-1028.

66 A. Rosenthal, A. Macdonald and J. Voldman, Biomaterials, 2007, 28, 3208-3216.

67 Y. S. Torisawa, B. H. Chueh, D. Huh, P. Ramamurthy, T. M. Roth, K. F. Barald and S. Takayama, Lab Chip, 2007, 7, 770-776.

68 A. Khademhosseini, J. Yeh, G. Eng, J. Karp, H. Kaji, J. Borenstein, O. C. Farokhzad and R. Langer, Lab Chip, 2005, 5, 1380-1386.

69 A. M. Skelley, O. Kirak, H. Suh, R. Jaenisch and J. Voldman, Nat. Methods, 2009, 6, 147-152.

70 M. Cordey, M. Limacher, S. Kobel, V. Taylor and M. P. Lutolf, Stem Cells, 2008, 26, 2586-2594.

71 B. Dykstra, J. Ramunas, D. Kent, L. McCaffrey, E. Szumsky, L. Kelly, K. Farn, A. Blaylock, C. Eaves and E. Jervis, Proc. Natl. Acad. Sci. U. S. A., 2006, 103, 8185-8190.

72 M. Lutolf, R. Doyonnas, K. Havenstrite, K. Koleckar and H. Blau, Integr. Biol., 2009, 1, 59-69.

73 I. Kurth, K. Franke, T. Pompe, M. Bornhaeuser and C. Werner, Integr. Biol., 2009, 1, 427-434.

74 L. A. Kunz-Schughart, J. P. Freyer, F. Hofstaedter and R. Ebner, J. Biomol. Screening, 2004, 9, 273-285.

75 X. Gidrol, B. Fouque, L. Ghenim, V. Haguet, N. Picollet-D'hahan and S. B, Curr. Opin. Pharmacol., 2009, 9, 664-668.

76 T. G. Fernandes, M. M. Diogo, D. S. Clark, J. S. Dordick and J. M. S. Cabral, Trends Biotechnol., 2009, 27. 342-349.

77 H. Otsuka, A. Hirano, Y. Nagasaki, T. Okano, Y. Horiike and K. Kataoka, ChemBioChem, 2004, 5, 850-855.

$78 \mathrm{~W}$. J. Wang, K. Itaka, S. Ohba, N. Nishiyama, U. I. Chung, Y. Yamasaki and K. Kataoka, Biomaterials, 2009, 30 , 2705-2715.

79 L. A. Kunz-Schughart, Cell Biol. Int., 1999, 23, 157-161.

$80 \mathrm{H}$. Yu, C. M. Alexander and D. J. Beebe, Lab Chip, 2007, 7, 388-391. 